Proceedings of the

Combustion

Institute

\title{
Effect of soot model, moment method, and chemical kinetics on soot formation in a model aircraft combustor
}

\author{
Shao Teng Chong ${ }^{\mathrm{a}, *}$, Venkat Raman ${ }^{\mathrm{a}}$, Michael E. Mueller ${ }^{\mathrm{b}}$, \\ Prabhu Selvaraj ${ }^{\mathrm{c}}$, Hong G. $\mathrm{Im}^{\mathrm{c}}$ \\ a Department of Aerospace Engineering, University of Michigan, Ann Arbor, MI 48109, USA \\ ${ }^{\mathrm{b}}$ Department of Mechanical and Aerospace Engineering, Princeton University, Princeton, NJ 08544, USA \\ ${ }^{\mathrm{c}}$ Clean Combustion Research Center, King Abdullah University of Science and Technology, Thuwal 23955, Saudi Arabia
}

Received 1 December 2017; accepted 12 June 2018

Available online xxx

\begin{abstract}
The simulation of turbulent sooting flames requires a host of models, of which the two critical components are the chemical kinetics that describe soot precursor evolution and the description of the soot population. The purpose of this study is to understand the sensitivity of soot predictions in a realistic aircraft combustor to model choices for these components. Two different chemistry mechanisms, three different statistical approaches, and two different soot inception models are considered. The simulations show that acetylene-based soot inception produces very high soot volume fraction, with the soot particles present predominantly in the inner recirculation zone of the swirl-stabilized combustor. The PAH-based nucleation models lead to soot generation in the shear layers emanating from fuel injection. The two advanced statistical approaches (Hybrid and Conditional Quadrature Method of Moments) also show significant differences. While the Hybrid method produces lower soot number density, it also generates larger soot particles due to a faster predicted rate of coagulation. The Conditional Quadrature approach produces much higher soot number density, but its particle sizes are smaller compared to the Hybrid method for all kinetic mechanisms considered. This experimental combustor is strongly dominated by surface growth based soot mass addition. As a result, even if nucleation/condensation rates are different, the final soot mass yield is comparable for PAH-based soot models. These results indicate the importance of not only the chemical mechanism, which may be less important in this surface growth dominated combustor, but also the soot statistical model, to which coagulation and the soot surface area are relatively sensitive.

(C) 2018 The Combustion Institute. Published by Elsevier Inc. All rights reserved.
\end{abstract}

Keywords: Method of moments; Soot chemistry; Pressurized combustor

\section{Introduction}

To achieve higher thermodynamic efficiency, gas

$*$ Corresponding author.

E-mail address: stchong@umich.edu (S.T. Chong). turbine combustors operate at increasingly higher pressures. Since soot formation is augmented by 
high pressures, there is a need to understand the formation and transport of soot within aerodynamically stabilized gas turbines [1]. Soot generation is intricately linked to gas-phase thermochemical conditions as well as the trajectories of the soot particles in the combustor $[2,3]$. Due to this inherent complexity, predictions of soot inside aircraft engines has been a formidable challenge. In particular, a host of models for describing the turbulent flow, the gas-phase combustion, and soot generation and evolution as well as the interactions of these processes are needed [2,4-7]. While there has been considerable progress in the modeling of soot formation processes $[8,9]$, the application of these advances to realistic combustor configurations still remain sparse. Previous studies [10-14] have shown that while the overall features of the flow can be captured rather accurately, prediction of soot volume fraction is highly sensitive to a host of conditions including small errors in flow field predictions and the choice of soot evolution models.

In modeling complex combustors, large eddy simulation (LES) has been shown to be accurate for predicting the reacting flow field $[2,14]$. With this basis for turbulence modeling, three additional components are required for modeling soot evolution: (a) a turbulent combustion model to describe the interactions between turbulence, gas-phase chemistry, and molecular transport; (b) a chemical kinetics model that links gas-phase combustion and soot precursor evolution to soot evolution through nucleation, condensation and surface growth; and (c) a soot population balance model and solution methodology that allows nanoparticles to be represented in a computationally tractable manner. Prior studies have shown that for flames that are not close to extinction, different combustion models produce comparable results $[15,16]$. Therefore, the focus here will be on the latter two modeling components mentioned above.

One of the main focus areas for soot research is the development of inception models that describe the transformation of gas-phase species into carbonaceous particles. While several models are available, the two main concepts are based on (a) an acetylene-based inception model $[4,17]$ and (b) a polycyclic-aromatic hydrocarbon $(\mathrm{PAH})$ based nucleation model [18-22]. Both models have been used extensively to simulate a variety of turbulent sooting flames $[5,7,23-26]$ with a range of predictive success. The semi-empirical acetylene-based inception mechanism produces very good reproduction of soot volume fraction for smaller hydrocarbon flames $[4,5,23]$ but vastly underpredicts inception for higher hydrocarbons such as kerosene [25]. Among the PAH-based nucleation models, those based on pyrene (A4) as soot precursor have often underpredicted the soot volume for ethylene and small-hydrocarbon flames $[15,26]$, while reasonable agreement was obtained for jet fuels [14].
On the other hand, recent nucleation models including higher PAHs up to coronene (A7) have shown improved soot prediction in laminar counterflow flames [21,22]. Moreover, the predictions were also found to be sensitive to the flow configuration used $[2,26]$. Therefore, it is of interest to investigate how the different soot chemistry models perform in turbulent flame simulations involving more complex flow-chemistry interactions.

Similarly, the description of the soot population can be achieved through multiple techniques $[2,12,27-29]$. In general, the soot population is described in terms of a number density function (NDF), which is a spatially and temporally varying function of certain internal coordinates that describe particle characteristics. In most cases, volume and surface area are chosen as the internal coordinates. The NDF is a high-dimensional function, spanning $N+4$ dimensions, where $N$ is the number of internal coordinates. For this reason, a direct solution of the NDF transport equation is not feasible. Statistical techniques can be classified into two groups for application in multidimensional unsteady simulations: (a) momentbased methods, where a set of moments of the NDF are defined and their transport equations are solved $[27,28,30]$, and (b) sectional methods, where the NDF is discretized into a finite number of sections in terms of the size class and transport equations solved for the number density in each of these sections. The latter approach is usually much more expensive, and an intractably large number of sections are needed for multi-variate distributions. Prior studies in laminar sooting flames have shown that there can be large variability in the results depending on the choice of moments and solution approach $[30,31]$. However, the effect of the choice on soot predictions has not been fully explored for turbulent sooting flames.

With this background, the goal of this study is to understand the sensitivity of soot predictions to the choice of chemistry model and moment approach for a model aircraft combustor. The study is based on the DLR ethylene/air combustor [32], which is comparable in design to a richquench-lean (RQL) type combustor used for aircraft propulsion. This combustor has also been studied using specific methods in the past [10-13], which will provide additional bases for comparison. Here, three different soot inception models and three different soot description approaches are compared with experimental data.

\section{Configuration and computational approach}

\subsection{DLR experimental combustor}

The model swirl combustor configuration used in this study is based on the high-pressure combustor of Geigle et al. [32]. A planar slice of the 

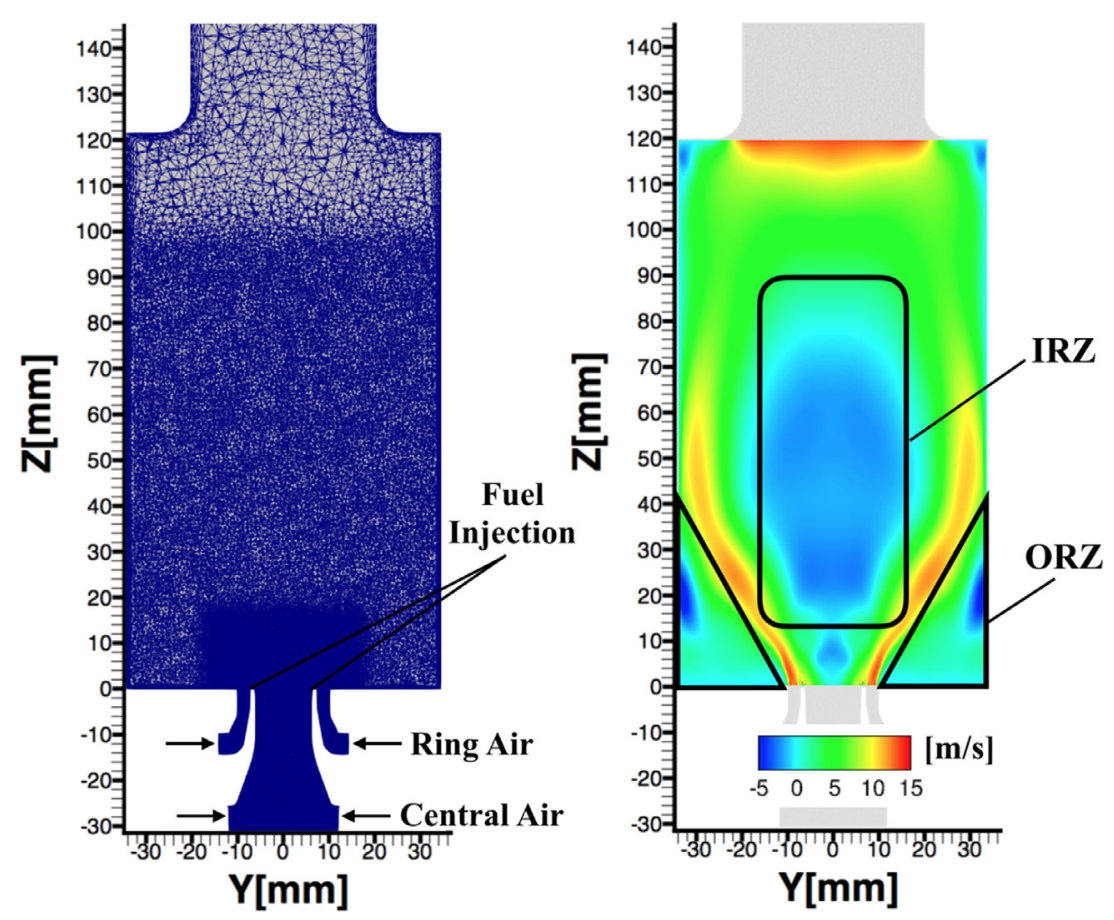

Fig. 1. (Left) DLR combustor geometry center cut-plane and mesh refinement with fuel and air inlets indicated. (Right) Time-averaged axial velocity with inner recirculation zone (IRZ) and outer recirculation zone (ORZ) indicated.

Table 1

Flow parameters and operating conditions for the simulated case. Flow rates referenced at 1.013 bar and $273 \mathrm{~K}$.

\begin{tabular}{lllll}
\hline $\begin{array}{l}\text { Pressure } \\
\text { (bar) }\end{array}$ & $\begin{array}{l}\mathrm{Q}_{\text {fuel }} \\
(\mathrm{slpm})\end{array}$ & $\begin{array}{l}\mathrm{Q}_{\text {central air }} \\
(\mathrm{slpm})\end{array}$ & $\begin{array}{l}\mathrm{Q}_{\text {ring air }} \\
(\mathrm{slpm})\end{array}$ & $\phi$ \\
\hline 3 & 39.3 & 140.8 & 328.5 & 1.2 \\
\hline
\end{tabular}

geometry and the computational mesh are shown in Fig. 1. A total of 12 million control volumes are present in the computational domain. The crosssectional area of the square combustor is $68 \times$ $68 \mathrm{~mm}^{2}$, with a height of $120 \mathrm{~mm}$. The inflow consists of three concentric nozzles: two air inlets with swirling velocity and 60 annular straight-channel fuel $\left(\mathrm{C}_{2} \mathrm{H}_{4}\right)$ inlets $\left(0.5 \times 0.4 \mathrm{~mm}^{2}\right.$ each), located in between the two air inlets. A constricted nozzle of diameter $40 \mathrm{~mm}$ serves as the combustor outlet.

In this study, the specific case in Table 1 is simulated. The global equivalence ratio is 1.2 , and the slight fuel rich condition could produce high volumes of soot if fuel-air mixing is inefficient. Therefore, the soot particles observed are generated due to local mixing inefficiencies. Further details about the configuration, computational domain, grid refinement process, and the statistical averaging process can be found in $[10,11,32,33]$.

\subsection{LES approach for sooting flames in complex geometries}

To simulate turbulent combustion, the LES approach is combined with the radiation flamelet/progress variable (RFPV) approach with a heat loss parameter, $H$, which accounts for radiation effects from the gas species and soot particles [26]. Further details on the implementation are provided in [10]. Transport equations for scalars (mixture fraction $Z$, progress variable $C$, heat loss parameter $H$, and PAH mass fraction $Y_{P A H}$ ) can be generically written as:

$\frac{\partial \rho \mathbf{q}}{\partial t}+[\nabla \cdot(\rho \mathbf{u})] \mathbf{q}=[\nabla \cdot(\rho D \nabla)] \mathbf{q}+\rho S(\mathbf{q})$,

where $\mathbf{q}=\left\{Z, C, H, Y_{P A H}\right\}$ is the solution vector. An explicit transport equation is solved for the lumped PAH mass fraction since its reaction time scales are much longer than that for fuel oxidation. Note that the constituent PAH species depend on the specific chemistry mechanism. The equations are filtered for LES, and the unclosed terms are modeled using the presumed subfilter PDF approach of Mueller and Pitsch [26]. Nonpremixed flamelet equations are solved, and the solutions stored in a flamelet table for lookup of the source terms, $S(\mathbf{q})=\left\{S_{Z}, S_{C}, S_{H}, S_{Y_{P A H}}\right\}$. These models are implemented in OpenFOAM [34], with modifications to ensure minimal energy dissipation and 
higher-order temporal accuracy [35]. This solver has previously been applied to simulating complex reacting flows $[10,36]$. Dynamic models were used for all subfilter quantities [37].

In this study, two variations in model components are considered: the chemical kinetics mechanism and the soot statistical description, the latter also having a variation in the soot inception model. The different models are described below.

\subsubsection{Chemical kinetics for soot formation}

The two chemistry mechanisms are selected based on the accuracy of predicting soot precursors: (a) Narayanaswamy et al. [19] (NBP hereinafter), and (b) KAUST-Aramco PAH Mech.1.0 [20] (KAM1.0 hereinafter). Both mechanisms include detailed PAH chemistry. The NBP mechanism includes PAH chemistry up to four aromatic rings (pyrene), and the KAM1.0 mechanism includes PAH chemistry up to seven aromatic rings (coronene). Both mechanisms have been validated against canonical laminar flames $[22,30,38]$.

\subsubsection{Soot statistical description}

To statistically model the soot NDF, three statistical approaches are considered, all based on methods of moments: (a) semi-empirical, (b) Hybrid Method of Moments (HMOM) and (c) Conditional Quadrature Method of Moments (CQMOM). The semi-empirical model, originally developed by Leung et al. [4], consists of an acetylene-based soot inception model. Transport equations are solved for only the soot mass and the soot number density, presumes that soot particles are spherical, and includes contributions from soot inception, coagulation, surface growth, and oxidation. Further details of the approach, including the rate expressions for inception, surface growth and oxidation can be found in $[4,5,39]$. The original rate coefficients in [4] are used in this study.

The HMOM and CQMOM methods solve for moments of the NDF and uses two internal coordinates, volume and surface area, to account for soot aggregate morphology. In the CQMOM approach $[40,41]$, the marginal NDF of volume is discretized using two delta functions, while the conditional NDF of surface area conditioned on volume coordinate is discretized using a single delta function. The moments of the NDF are then written as [40]:

$$
M_{x, y}=\sum_{i=1}^{N_{v}} \sum_{j=1}^{N_{a}} \omega_{i} \omega_{i j} V_{i}^{x} S_{i j}^{y},
$$

where $N_{v}$ and $N_{a}$ are number of volume and surface area nodes, respectively. For this study, $N_{v}=2$ and $N_{a}=1$. Using the moment-inversion algorithm, $\omega_{i}$ and $\omega_{i j}$ are obtained at each computational grid point at each time step. The set of moments used for this study are: $M_{0,0}, M_{1,0}, M_{2,0}, M_{3,0}, M_{0,1}$, $M_{1,1}$. In the CQMOM approach, the locations in coordinate space (i.e., $V_{i}$ and $S_{i j}$ ) are determined exclusively based on the local set of moments.

In the HMOM method [30], small incipient particles are described with a quadrature-approach not dissimilar to CQMOM, but the large particles are described with polynomial interpolation, similar to
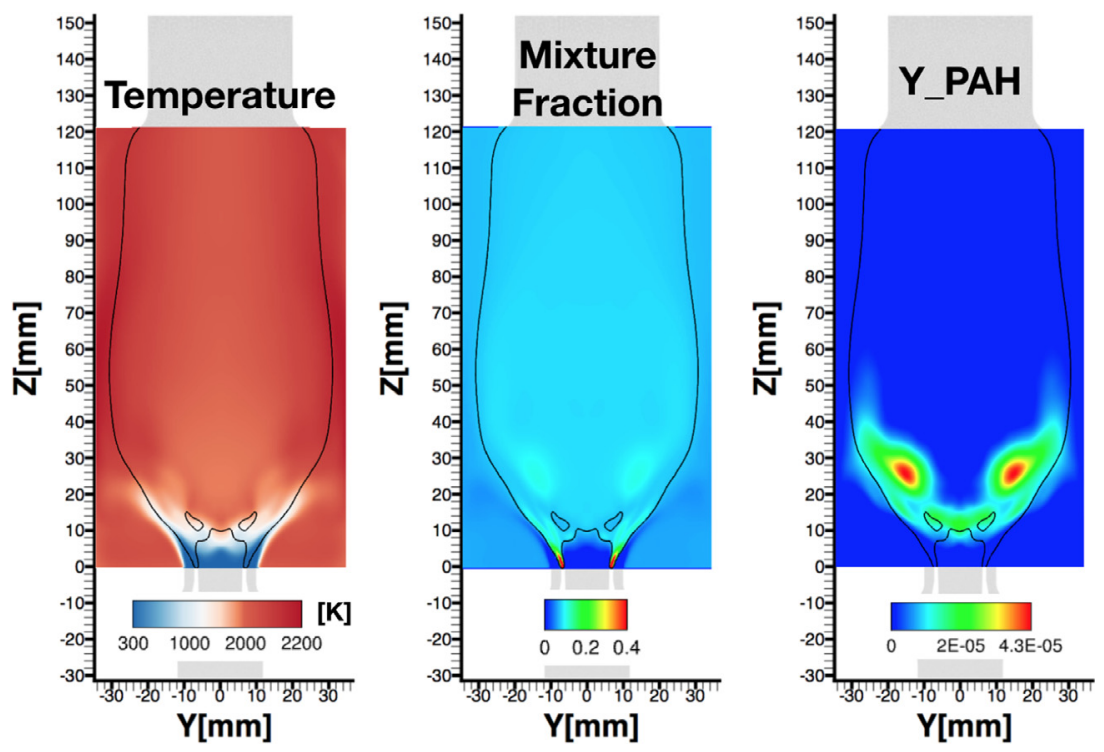

Fig. 2. Time averaged temperature, mixture fraction, and PAH mass fractions, lined with stoichiometric mixture fraction for the NBP mechanism and CQMOM model. 

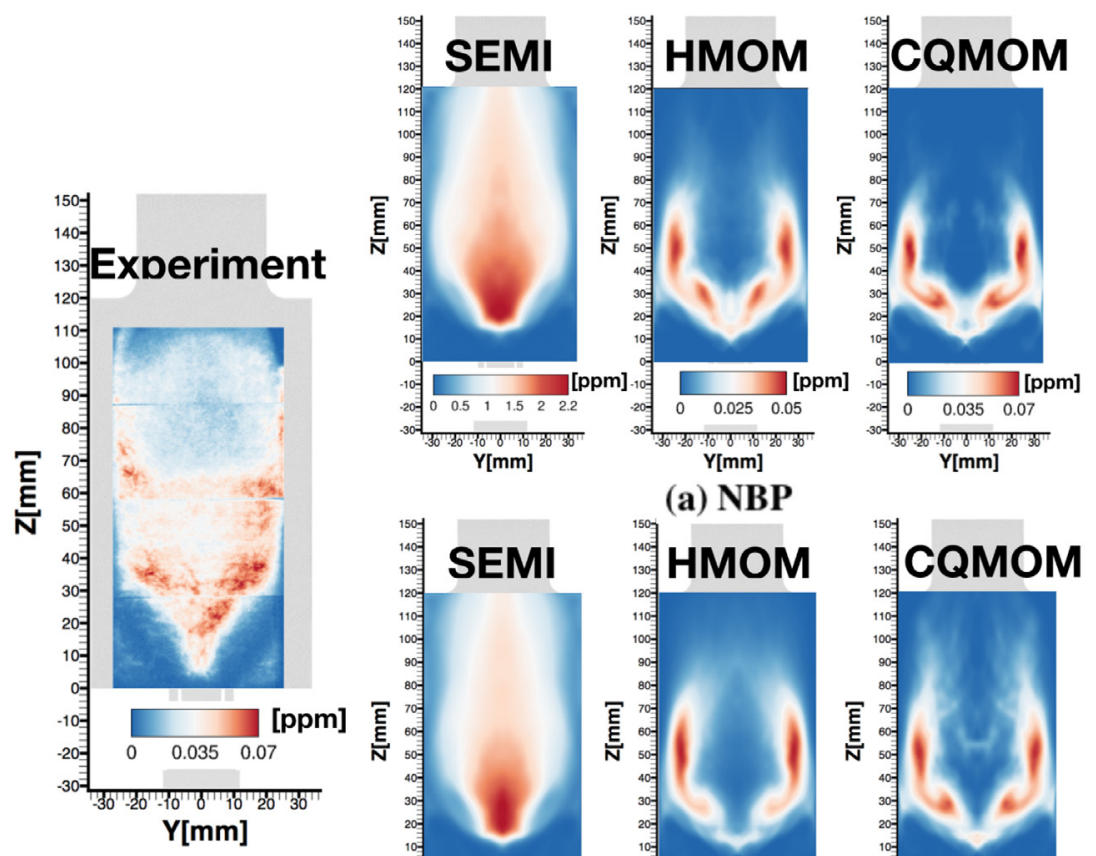

(a) NBP
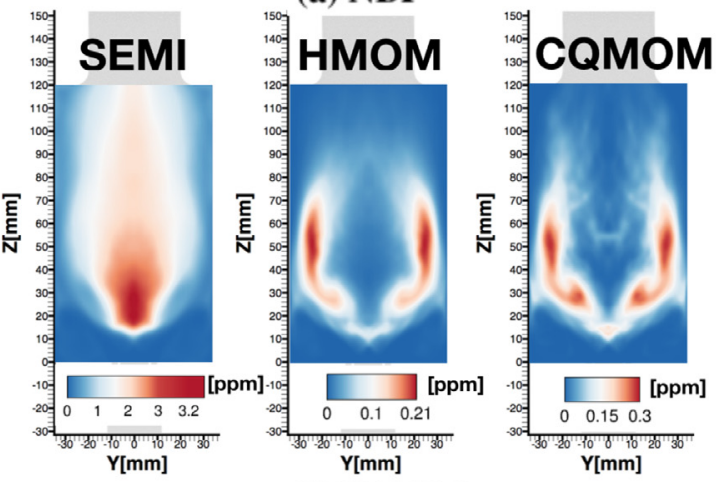

(b) KAM1.0

Fig. 3. Time-averaged soot volume fraction contours for the experimental measurement (left) and the different soot and moment description models.

earlier moment methods [27]:

$$
M_{x, y}=\omega_{0} V_{0}^{x} S_{0}^{y}+\exp \left(\sum_{r=0}^{R} \sum_{k=0}^{r} a_{r, k} x^{k} y^{r-k}\right),
$$

where $\omega_{0}, V_{0}$, and $S_{0}$ are the weight, volume, and surface area of the incipient particles, respectively. An additional equation is solved for $\omega_{0}$ in addition to 3 moments for the larger particles: $M_{0,0}, M_{1,0}$, $M_{0,1}$.

For both the HMOM and CQMOM approaches, the source terms for nucleation are obtained based on PAH concentration as imposed by the chemistry mechanisms. The HACA mechanism [42] is used to describe soot growth, and other terms related to PAH condensation, coagulation, and oxidation are detailed in [30].

\subsection{Simulation cases}

Based on the above description, six different simulations were conducted for the DLR combustor operating at 3 bar pressure. A set of three simulations using semi-empirical, HMOM, and CQMOM approaches for the NBP mechanism and another set of the same statistical models with the KAM1.0 mechanism. The simulations were run for
20 flow-through times until the soot statistics converged. The source terms for the semi-empirical model were based on the acetylene concentration from the individual kinetic mechanisms used. A flamelet library with necessary species mass fractions and source terms was generated for each mechanism and tabulated in $\widetilde{Z}, \widetilde{C}, \widetilde{Z^{\prime \prime 2}}, \widetilde{H}$ coordinates, where $\sim$ refers to the LES filtering operation and $\widetilde{Z^{\prime \prime 2}}$ refers to the subfilter variance of mixture fraction.

\section{Results and discussion}

Figure 2 shows the time-averaged temperature, mixture fraction, and $\mathrm{PAH}$ concentration for the CQMOM method using the NBP mechanism. Due to the strong swirling inflow, fuel-air mixing is nearly complete within a short distance downstream of the fuel injection ports. Consequently, the temperature field is nearly uniform beyond $Z=$ $30 \mathrm{~mm}$. The mixture fraction field shows the presence of two shear layers, with high mixture fraction values confined to these regions that denote the boundaries of the IRZ and ORZ in Fig. 1. Moreover, there are small regions of rich mixture 

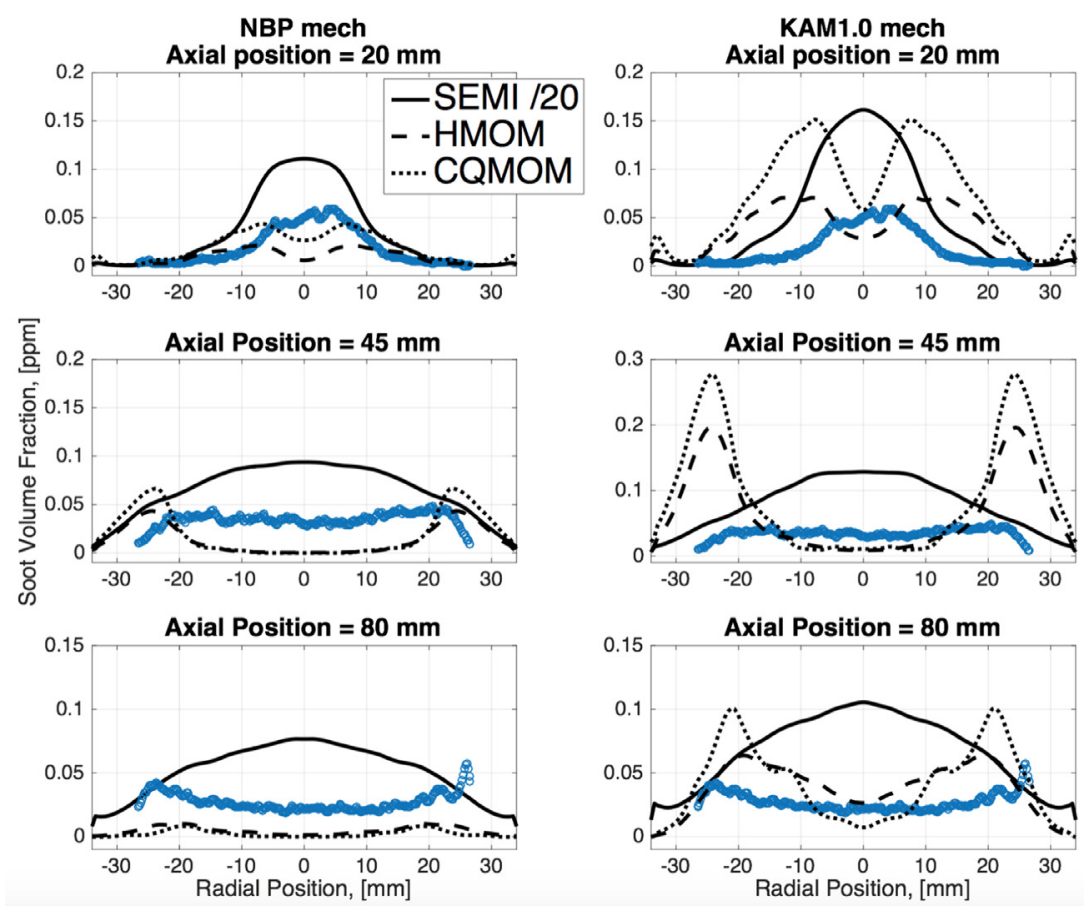

Fig. 4. Comparisons of time-averaged soot volume fraction with experimental data (blue scatter) for all mechanisms and soot models, at various axial positions in the combustor. (For interpretation of the references to color in this figure legend, the reader is referred to the web version of this article.)

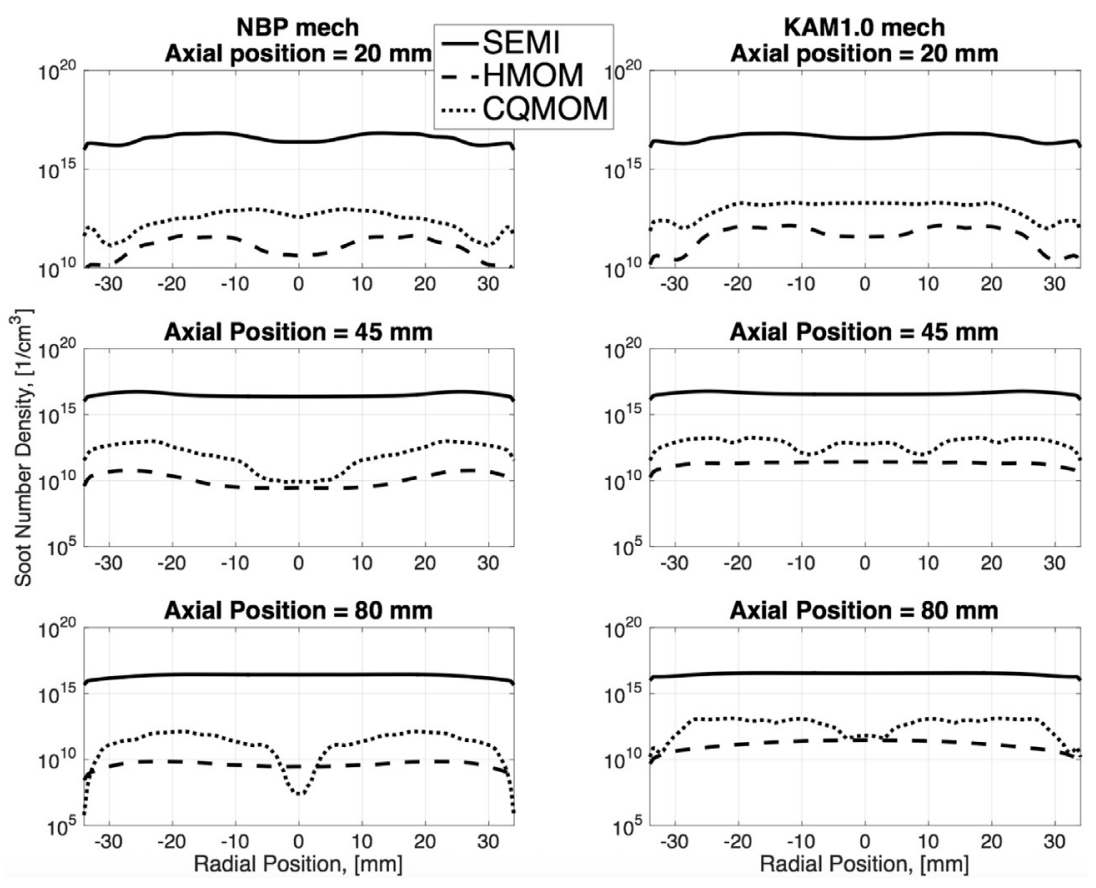

Fig. 5. Comparisons of time-averaged soot number density for all mechanisms and soot models, at various axial positions in the combustor. 

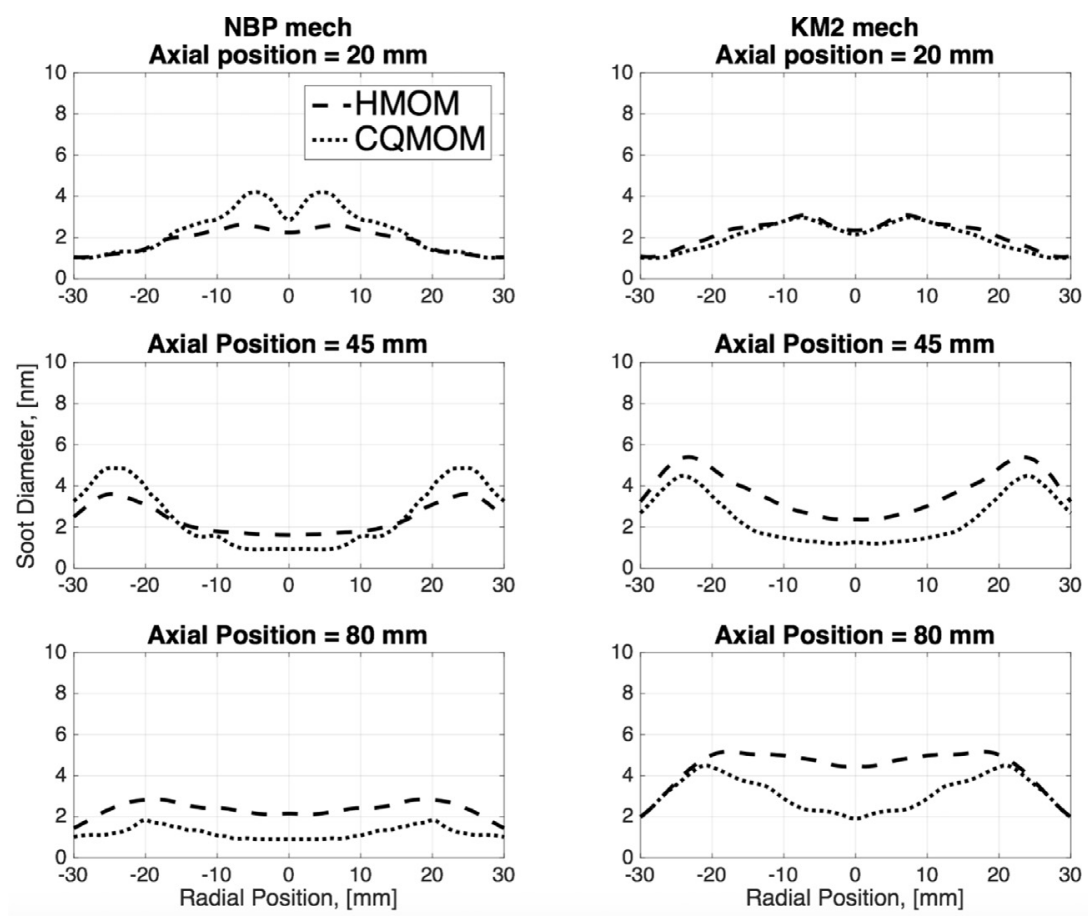

Fig. 6. Comparison of time-averaged soot primary particle diameter for HMOM and CQMOM models at various axial positions in the combustor.

fraction further downstream caused by the recirculating flow in the IRZ. The PAH concentrations peak at this location but form a broad high value zone near the base of the combustor. Overall, the agreement for flow field velocity and gas-phase thermodynamic quantities are very good, and has been studied extensively in prior work [10].

The key results of the time-averaged soot volume fraction and number density are shown in Figs. 3-5. Several observations are noted. The semiempirical model, with an acetylene-based inception model, predicts much of the soot mass to be in the IRZ, which tends to be slightly fuel rich for this case and supports large concentrations of acetylene but little PAH. Similar large soot volume fractions in the IRZ have been found by Katta et al. [43] using a semi-empirical soot model for a swirl combustor at $\phi=1.15$ and JP-8 fuel. In the IRZ, the semi-empirical model predicts an approximately constant level of soot volume fraction. Conversely, the other soot models utilize PAH-based inception and predict soot formation primarily in the shear layers that separate the IRZ and ORZ. The fuel jets that enter from the annular ring provide this extremely fuel-rich region that supports large $\mathrm{PAH}$ concentrations. In addition, since PAH is highly sensitive to strain rate [3], the soot formation in this shear layer exhibits high intermittency due to turbulence-induced fluctuations in the local strain rate $[2,10,11]$. The soot models differ in the growth pathways and their dependence on mixture fraction. Hence, if the mixture fraction, for which there is no experimental data, is incorrect in the IRZ, then the soot volume fraction could be predicted incorrectly. The oxidation rates are unmodified from values in the literature for both the SEMI and detailed models. However, the presumed subfilter PDF model used with the detailed chemistry model could also lead to overprediction of the oxidation rate in the IRZ [44].

Quantitative comparisons with experimental data are shown in Fig. 4, where the radial distribution of the soot volume fraction is shown at three different axial locations. It is evident that the three models yield significant differences. At upstream locations, the experiments show a peak in soot volume in the IRZ, which is consistent with the semi-empirical model, although it is overpredicted by an order of magnitude. At downstream locations, soot peaks are shifted further to the shear layer regions, which is qualitatively reproduced by the PAH-based models. Between the two detailed soot chemistry mechanisms, the use of the KAM1.0 mechanism increases soot volume fraction by approximately a factor of four. However, neither chemical model agrees with the experimental measurements any better than the other, but the KAM1.0 model does provide persistent soot 

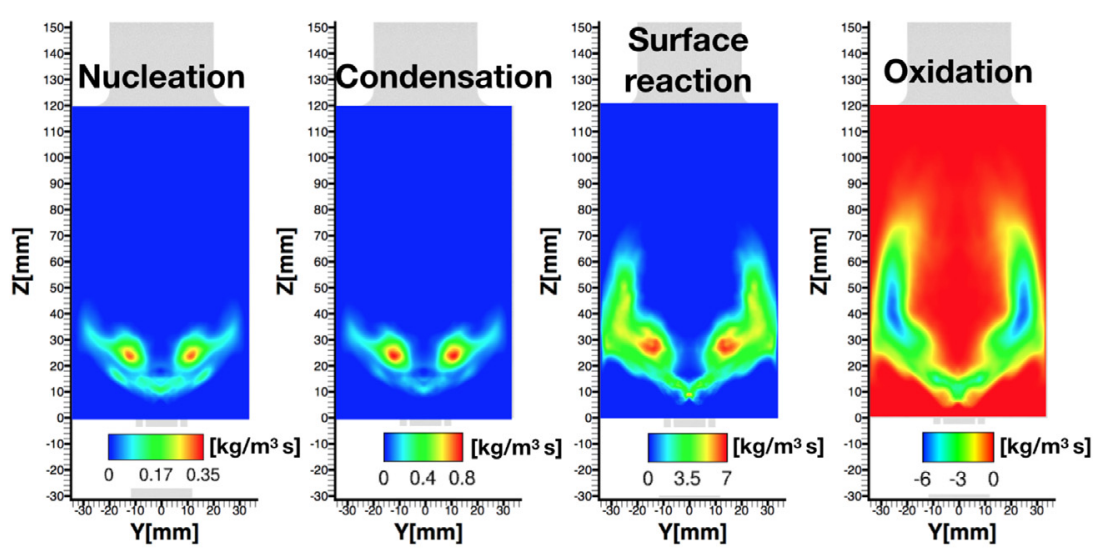

(a) CQMOM/NBP
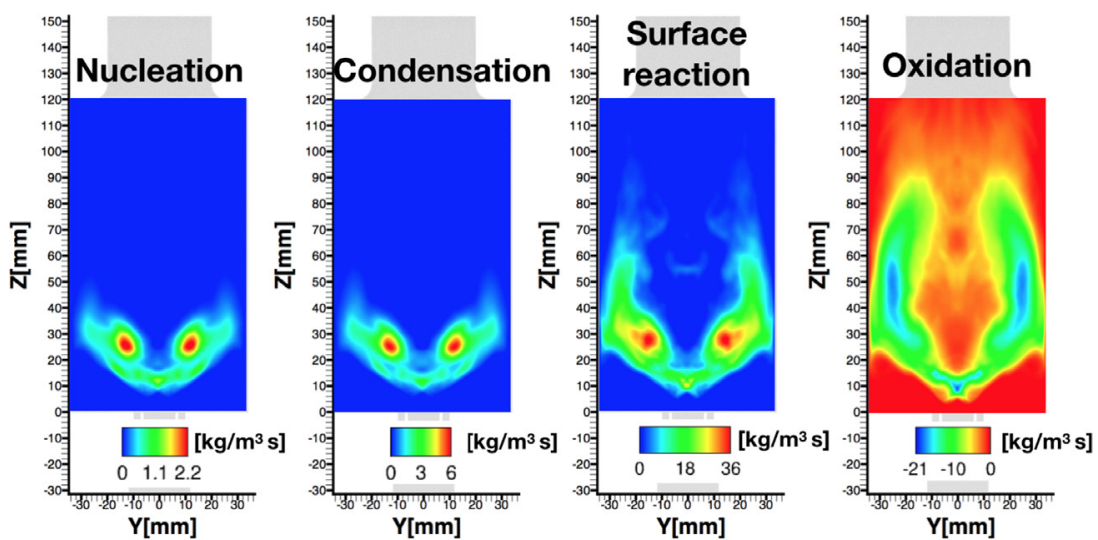

(b) CQMOM/KAM1.0

Fig. 7. Time-averaged soot source terms using CQMOM approach for the the NBP and KAM1.0 mechanisms.

further downstream compared to the NBP model in agreement with the experimental measurements. CQMOM predicts soot volume fractions up to twice as large as HMOM, especially upstream.

As shown in Fig. 3, HMOM and CQMOM produce qualitatively similar soot volume fractions with either mechanism, differing by up to a factor of two. This can be better understood through the number density contours shown in Fig. 5. CQMOM predicts number density significantly larger than HMOM. Since the soot inception models are the same, the only explanation for the difference in number density is due to coagulation, with HMOM predicting a faster rate of coagulation. Since coagulation reduces the surface area [30], HMOM has less surface area, less surface growth, and subsequently a smaller volume fraction compared to CQMOM. The semi-empirical model predicts a considerably larger number density than either HMOM or CQMOM consistent with the volume fraction. This indicates that the soot inception rate is much larger for the acetylene-based inception model than the PAH-based inception model.

Figure 6 shows the comparison of the primary particle diameter for the CQMOM and HMOM models at two different axial locations in the combustor and confirms the observations above. The average primary particle diameter is considerably larger with HMOM, indicating an increased rate of coagulation. This is independent of the chemical mechanism used.

The change in the chemical mechanism from NBP to KAM1.0 consistently increases soot volume fraction for all the soot models considered, consistent with previous studies in laminar flames [22]. While the qualitative volume fraction profiles are the same, there is $50-200 \%$ increase in soot volume fraction. This is consistent with the prediction of acetylene $\left(\mathrm{C}_{2} \mathrm{H}_{2}\right)$ concentration and 
the PAH concentrations, which are higher with KAM1.0 compared to the NBP mechanism. The difference is due presumably to the base chemistry, with KAM1.0 validated for only $\mathrm{C}_{0}-\mathrm{C}_{2}$ and NBP for $\mathrm{C}_{0}-\mathrm{C}_{8}$. In addition to the base chemistry, the PAH growth pathways also vary between the mechanisms. In KAM1.0, PAH growth continues beyond pyrene and the growth is largely due to acetylene addition to PAH radicals. In NBP, PAH growth stops at pyrene but includes more detailed pathways including the effects of $C_{3}$ (propargyl), $C_{5}$ (cyclopentadyenyl), $C_{7}$ (benzyl), and $C_{9}$ (indenyl) radical species.

To conclude the discussion of these differences, the source terms for the volume moment $M_{1,0}$ are plotted in Fig. 7 for the CQMOM simulations. As expected, nucleation dominates near the base of the flow, in the region adjacent to the shear layers within the IRZ. Condensation is similarly distributed. However, surface growth is the most dominant source of soot mass, extending from the shear layer into the IRZ. In fact, even near the base of the flow, higher acetylene concentrations lead to increased surface growth rates. Oxidation is roughly collocated with the surface growth region, on the fuel-lean side of the stoichiometric location that cuts across the shear layer. The two kinetic mechanisms produce vastly different nucleation and condensation rates, differing by an order of magnitude at certain locations. Despite this difference, the dominant role of surface growth reduces the disparity in the final soot volume fractions generated by these mechanisms (Fig. 3). Furthermore, the dominance of surface growth, which depends strongly on the soot surface area and coagulation dynamics, indicates that coagulation can be extremely important for dictating the soot volume fraction in turbulent combustion. Therefore, highfidelity closures for soot-turbulence interactions are required and will have leading-order impacts on the soot volume fraction.

\section{Conclusions}

LES computations to understand effects of the chemical mechanism and soot statistical model on soot predictions were conducted. Two different kinetic mechanisms (NBP, KAM1.0) and three different statistical approaches (semi-empirical, HMOM, CQMOM) were considered. The simulations yielded the following conclusions for the models considered.

- The use of acetylene-based inceptions leads to significantly higher soot volume fraction compared to the experiments or the PAHbased inception models. However, this approach also generates significant soot mass in the IRZ of the swirl combustor, which is consistent with the experimental data.
The PAH-based nucleation models tend to generate soot in the shear layers, where rapid changes in strain rate lead to intermittency in soot inceptions.

- The different statistical approaches generate different soot number density, indicating significant differences in the handling of the NDF. The semi-empirical model produced very high number density and soot mass yield. The CQMOM simulations show significantly higher number density compared to HMOM, although the final soot yield is comparable. The CQMOM particle diameter is correspondingly smaller, and the difference is attributed to a faster rate of coagulation predicted with HMOM.

- The KAM1.0 mechanism produces more soot mass compared to the NBP mechanism for all statistical approaches considered, which is consistent with laminar flame results [22]. While the nucleation and condensation source terms are much higher with KAM1.0 compared to NBP, the final soot mass fraction changes by less than a factor of two. In the combustor, surface growth through acetylene-driven HACA mechanism dominates over other processes such as nucleation or condensation in adding soot mass. As a result, even with the large variations in these rates for the two kinetic mechanisms, the final soot yields are comparable. However, the dominance of surface growth accentuates differences in the coagulation rates predicted by the statistical models.

This study demonstrates that soot predictions are sensitive to the choice of all models involved, and care must be taken in interpreting the results. From an uncertainty standpoint, the changes in soot predictions with such model changes should be incorporated [45]. Improvements to the statistical approaches that can ensure numerical convergence of the moments approach will be useful for ensuring reliability of the predictions. In this sense, other solution techniques [28,29] show promise and should be further investigated. From a physics standpoint, the performance of such models in combustors where nucleation/condensation dominate soot mass would be interesting, and the hypothesis is that differences due to chemical mechanisms would be larger but differences due to statistical models smaller.

\section{Acknowledgments}

The authors gratefully acknowledge financial support from NASA through grant NNX16AP90A with Dr. Jeff Moder as program monitor. STC and VR were partially supported by grant 1975-08 
CCF CCRC from KAUST. HGI and PS were supported by competitive funding from KAUST.

\section{References}

[1] A.E. Karatas, Ö.L. Gülder, Prog. Energ. Combust. Sci. 38 (2) (2012) 818-845.

[2] V. Raman, R.O. Fox, Ann. Rev. Fluid Mech. 48 (2016) 159-190.

[3] F. Bisetti, G. Blanquart, M.E. Mueller, H. Pitsch, Combust. Flame 159 (1) (2012) 317-335.

[4] K.M. Leung, R.P. Lindstedt, W. Jones, Combust. Flame 87 (3-4) (1991) 289-305.

[5] M. Fairweather, W. Jones, H. Ledin, R. Lindstedt, in: Symp. Int. Combust., vol. 24, Elsevier, 1992, pp. 1067-1074.

[6] I.M. Kennedy, Prog. Energ. Combust. Sci. 23 (2) (1997) 95-132.

[7] H. El-Asrag, S. Menon, Combust. Flame 156 (2) (2009) 385-395.

[8] H. Wang, M. Frenklach, Combust. flame 110 (1) (1997) 173-221.

[9] H. Bockhorn, Soot Formation in Combustion: Mechanisms and Models, vol. 59, Springer Science \& Business Media, 2013.

[10] S.T. Chong, M. Hassanaly, H. Koo, M.E. Mueller, V. Raman, K.-P. Geigle, Combust. Flame 192 (2018) 452-472.

[11] H. Koo, M. Hassanaly, V. Raman, M.E. Mueller, K.P. Geigle, J. Eng. Gas Turbines Power 139 (3) (2017) 031503.

[12] C. Eberle, P.M. Gerlinger, K.P. Geigle, M. Aigner, in: 50th AIAA/ASME/SAE/ASEE Joint Propulsion Conference, 2014 2014-3472.

[13] A. Wick, F. Priesack, H. Pitsch, in: Proceedings of the ASME Turbo Expo 2017: Turbomachinery Technical Conference \& Exposition, 2017, pp. 1-10.

[14] M.E. Mueller, H. Pitsch, Phys. Fluids 25 (11) (2013) 110812

[15] P. Donde, V. Raman, M.E. Mueller, H. Pitsch, Proc. Combust. Inst. 34 (2013) 1183-1192.

[16] V. Raman, H. Pitsch, Combust. Flame 142 (4) (2005) 329-347.

[17] J. Moss, C. Stewart, K. Young, Combust. Flame 101 (4) (1995) 491-500.

[18] M. Saffaripour, A. Veshkini, M. Kholghy, M.J. Thomson, Combust. Flame 161 (3) (2014) 848-863.

[19] K. Narayanaswamy, G. Blanquart, H. Pitsch, Combust. Flame 157 (10) (2010) 1879-1898.

[20] W.K. Metcalfe, S.M. Burke, S.S. Ahmed, H.J. Curran, Int. J. Chem. Kinet 45 (10) (2013) 638-675.

[21] Y. Wang, A. Raj, S. Chung, Combust. Flame 162 (2015) 586-596.
[22] P. Selvaraj, P.G. Arias, B.J. Lee, et al., Combust. Flame 163 (2016) 427-436.

[23] A. Kronenburg, R. Bilger, J. Kent, Combust. Flame 121 (1) (2000) $24-40$.

[24] G. Blanquart, H. Pitsch, Combust. Flame 156 (8) (2009) 1614-1626.

[25] Z. Wen, S. Yun, M. Thomson, M. Lightstone, Combust. Flame 135 (3) (2003) 323-340.

[26] M.E. Mueller, H. Pitsch, Combust. Flame 159 (2012) 2166-2180.

[27] M. Frenklach, Chem. Eng. Sci. 57 (12) (2002) 2229-2239.

[28] D.L. Marchisio, R.O. Fox, Computational Models for Polydisperse Particulate and Multiphase Systems, Cambridge University Press, 2013.

[29] F. Sewerin, S. Rigopoulos, Phys. Fluids 29 (10) (2017) 105105.

[30] M.E. Mueller, G. Blanquart, H. Pitsch, Combust. Flame 156 (2009) 1143-1155.

[31] A. Zucca, D.L. Marchisio, M. Vanni, A.A. Barresi, AIChE J. 53 (4) (2007) 918-931.

[32] K.P. Geigle, M. Köhler, W. OLoughlin, W. Meier, Proc. Combust. Inst. 35 (3) (2015) 3373-3380.

[33] S.T. Chong, V. Raman, M.E. Mueller, H.G. Im, in: Proceedings of the ASME Turbo Expo 2018: Turbomachinery Technical Conference \& Exposition, 2018, pp. 1-9.

[34] The open source cfd toolbox (2017). OpenCFD Inc. http://www.openfoam.com.

[35] M. Hassanaly, H. Koo, C.F. Lietz, S.T. Chong, V. Raman, Comput. Fluids 162 (2018) 11-25.

[36] H. Koo, M. Hassanaly, V. Raman, M.E. Mueller, K.P. Geigle, in: Proceedings of the ASME Turbo Expo 2016: Turbomachinery Technical Conference \& Exposition, 2016, pp. 1-10.

[37] P. Moin, K. Squires, W. Cabot, S. Lee, Phys. Fluids A 3 (1991) 2746-2757.

[38] G. Blanquart, P. Pepiot-Desjardins, H. Pitsch, Combust. Flame 156 (3) (2009) 588-607.

[39] A. Kronenburg, R. Bilger, J. Kent, Combust. Flame $121(1-2)(2000) 24-40$.

[40] C. Yuan, R.O. Fox, J. Comput. Phys. 230 (22) (2011) 8216-8246.

[41] Y. Sung, V. Raman, H. Koo, M. Mehta, R.O. Fox, AIChE J. 60 (2) (2014) 459-472.

[42] M. Frenklach, H. Wang, in: Symp. Int. Combust., vol. 23, Elsevier, 1991, pp. 1559-1566.

[43] V.R. Katta, T.R. Meyer, C. Montgomery, W.M. Roquemore, in: 41th AIAA/ASME/SAE/ASEE Joint Propulsion Conference and Exhibit, 2005. no. 2005-3777.

[44] International sooting ame workshop 32016.

[45] M.E. Mueller, V. Raman, Combust. Flame 161 (7) (2014) 1842-1848. 